\title{
Black Hole Production in Particle Collisions and Higher Curvature Gravity
}

\author{
Vyacheslav S. Rychkov* \\ Insituut voor Theoretische Fysica, Universiteit van Amsterdam \\ Valckenierstraat 65, 1018XE Amsterdam, The Netherlands
}

(Dated: April 2004)

\begin{abstract}
The problem of black hole production in transplanckian particle collisions is revisited, in the context of large extra dimensions scenarios of $\mathrm{TeV}$-scale gravity. The validity of the standard description of this process (two colliding Aichelburg-Sexl shock waves in classical Einstein gravity) is questioned. It is observed that the classical spacetime has large curvature along the transverse collision plane, as signaled by the curvature invariant $\left(R_{\mu \nu \lambda \sigma}\right)^{2}$. Thus quantum gravity effects, and in particular higher curvature corrections to the Einstein gravity, cannot be ignored. To give a specific example of what may happen, the collision is re-analyzed in the Einstein-Lanczos-Lovelock gravity theory, which modifies the Einstein-Hilbert Lagrangian by adding a particular 'GaussBonnet' combination of curvature squared terms. The analysis uses a series of approximations, which reduce the field equations to a tractable second order nonlinear PDE of the Monge-Ampère type. It is found that the resulting spacetime is significantly different from the pure Einstein case in the future of the transverse collision plane. These considerations cast serious doubts on the geometric cross section estimate, which is based on the classical Einstein gravity description of the black hole production process.
\end{abstract}

PACS numbers: 04.70.-s, 04.50.+h, 11.10Kk

*Electronic address: rychkov@science.uva.nl 


\section{INTRODUCTION}

All present-day macroscopic experimental data are consistent with gravity being described by the 4-dimensional Einstein-Hilbert action

$$
S=-\frac{1}{16 \pi G} \int R \sqrt{-g} d^{4} x
$$

In the microworld no gravitational effects have been observed, and indeed the 4-dimensional (4d) Newton constant $G$ in (10) is so small that such effects would be totally negligible up to unreachable collider energies of $M_{\mathrm{Pl}}^{(4)} \sim 10^{19} \mathrm{GeV}$ (the $4 \mathrm{~d}$ Planck scale).

The idea of large extra dimensions allows to imagine a world in which gravity is strong enough to play a role in elementary particle collisions at accessible energies. In this world Einstein gravity propagates in $D>4$ dimensions, out of which $D-4$ are curled up in a compact manifold of size $\ell \gg 1 / M_{\mathrm{Pl}}^{(4)}$. At distances $\gg \ell$ such gravity would be described by an effective $4 \mathrm{~d}$ action of the form (11). To get the effective $4 \mathrm{~d}$ Newton constant right, we have to set the $D$-dimensional Planck scale at [1]

$$
M_{\mathrm{Pl}}^{(D)} \sim M_{\mathrm{Pl}}^{(4)}\left(M_{\mathrm{Pl}}^{(4)} \ell\right)^{-\frac{D-4}{D-2}} \ll M_{\mathrm{Pl}}^{(4)}
$$

This means that such gravity will be much stronger than we are used to believe at short distances $\ll \ell$.

Motivated by the hierarchy problem, Arkani-Hamed, Dimopoulos and Dvali 1] proposed to push this idea to the extreme and lower $M_{\mathrm{Pl}}$ all the way down to the electroweak scale $M_{\mathrm{EW}} \sim 1 \mathrm{TeV}$. This proposal requires extra dimensions ranging from a mm to a fermi for $6 \leq D \leq 11$ ( $D=5$ leads to $\ell \sim 10^{13} \mathrm{~cm}$ and is excluded $)$. To make this consistent with the 4d Standard Model, we have to assume that all the other fields but gravity do not feel the extra dimensions. They must be confined to a $4 \mathrm{~d}$ submanifold (brane) of the $D$-dimensional world (bulk).

The simplest collider signature of such braneworld scenarios would be apparent energy non-conservation due to produced gravitons escaping into the bulk. Recent Tevatron

searches [2] of such missing energy events found no statistically significant effect and set a lower bound of $\sim 0.6 \mathrm{TeV}$ on the $D$-dimensional Planck scale (for a general review of signals and constraints, see [3]). Of course the LHC with its c.m. energy of $14 \mathrm{TeV}$ will be a much better probe of such phenomena. 
In this paper I would like to contribute to the unfinished discussion of another possible signature of $\mathrm{TeV}$-scale gravity — black hole production in transplanckian collisions (see reviews [4]).

The common current opinion is that this process may be adequately described using classical general relativity, and that a single large black hole will form for a range of impact parameters (in the $D$-dimensional Planck units)

$$
b \lesssim R_{\text {Schw }}(E) \sim E^{1 /(D-3)} .
$$

Here $E \gg 1$ is the energy of colliding particles, and $R_{\text {Schw }}(E)$ is the Schwarzschild radius of the $D$-dimensional static black hole of mass $E$. If production cross section

$$
\sigma \sim \pi\left[R_{\mathrm{Schw}}(E)\right]^{2}
$$

based on this estimate (known as the geometric cross section) were true, the LHC would produce black holes at a rate $\sim 1 \mathrm{~Hz}$ for $M_{\mathrm{Pl}}=1 \mathrm{TeV}$, becoming a black hole factory [5].

In older studies of transplanckian collisions [6] the authors usually carefully stated that in the range (3) a black hole is expected to form. Indeed, the eikonal expansion, normally used to analyze quantum gravity in the transplanckian regime, breaks down exactly in that range. It seems that the geometric cross section proposal was first put forward unequivocally by Banks and Fischler [7].

Later on, an appealing theoretical argument backing (3), (44) was proposed by Eardley and Giddings [8]. In the first step of the argument the problem of black hole formation is analyzed in classical Einstein gravity using the closed trapped surface (CTS) method. A welldefined mathematical problem of finding a CTS in the spacetime formed by superposing two Aichelburg-Sexl shock waves is formulated and solved. For the range of impact parameters when a CTS is found, black hole formation is concluded by invoking the Cosmic Censorship Conjecture.

In the second step the aim is to argue that quantum corrections to general relativity are not likely to modify the conclusions, because the classical spacetime has small curvature in the regions relevant for the trapped surface evolution. However, it is this second step where I disagree with Eardley and Giddings. ${ }^{1}$

\footnotetext{
${ }^{1}$ It should be mentioned that the validity of the geometric cross section was also challenged by Voloshin 9]. Some of his criticisms were addressed in [8, 10], others still remain unanswered. There does not seem to be an obvious connection between Voloshin's and my reasons for critique.
} 
As we will see, curvature becomes large on the transverse plane at the moment when the particles pass each other, and in the future of this plane corrections to Einstein gravity cannot be ignored. In particular, if higher order curvature terms are present in the effective gravitational Lagrangian (and we have no reason to believe that they don't), they will become important precisely at this moment.

To see a specific example of how this might happen, we will add to the Einstein-Hilbert Lagrangian a particular combination of curvature squared terms $\left(R_{\mu \nu \lambda \sigma}\right)^{2}-4\left(R_{\mu \nu}\right)^{2}+R^{2}$ first considered in $D$ dimensions by Lovelock [11], with a coefficient of natural magnitude $\sim 1$ in Planck units. The technical reason why I choose this combination is that it leads to second order equations of motion. My primary goal in this paper is to furnish an example of how things may go wrong, and it is unlikely that any other combination would be better in this respect.

In this Einstein-Lovelock theory we will then consider the simplest - zero impact parameter - collision case. Existence of a CTS for such head-on collisions was shown long ago by Penrose [12]. We will see that the curvature squared terms indeed become important and significantly modify the spacetime to the future of the transverse collision plane.
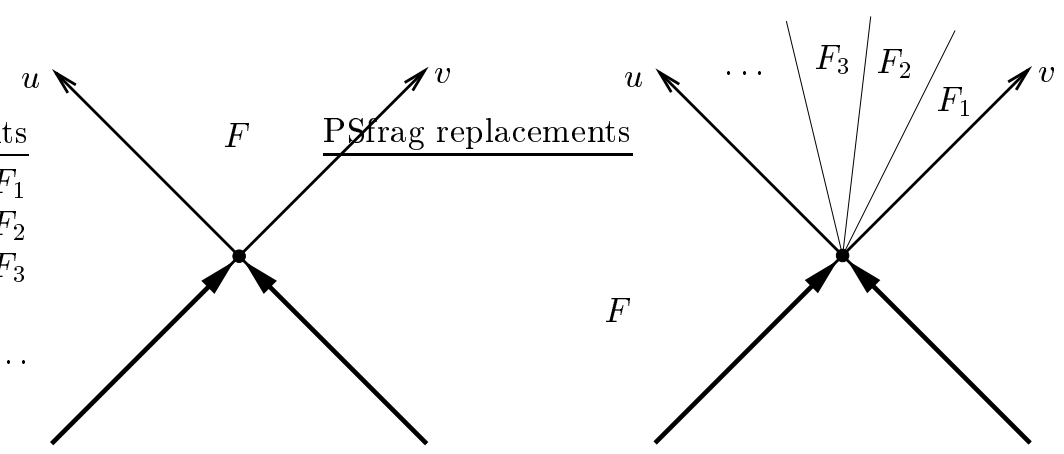

FIG. 1: The longitudinal slice of the collision spacetime in the pure Einstein and the EinsteinLovelock theories

The result of this analysis is summarized in Fig. 1. In the Einstein case the post-collision spacetime is weakly curved (at large transverse radii). The metric in the future wedge $F$ can be expanded in a power series in the light-cone coordinates $u, v$, starting with a linear term $\propto u+v$. The Riemann tensor has $\delta$-function singularities on the null planes $u=0$ and $v=0$; the Ricci tensor is of course everywhere zero. That curvature becomes large can be seen from the invariant $\left(R_{\mu \nu \lambda \sigma}\right)^{2} \propto \delta(u) \delta(v)$. 
On the contrary, in the Einstein-Lovelock case the future wedge is split into sectors $F_{1}, \ldots, F_{n}, n \geq 2$, separated by planes on which the Riemann tensor has additional $\delta$ function singularities. Moreover, the Ricci tensor is also singular on these planes, and in general nonzero in between. In particular, the post-collision spacetime does not satisfy Einstein's equations. An additional feature is that the number of sectors $n$ is arbitrary; the solution is not unique.

The paper thus contains two main results - a general observation (curvature becomes large) and a specific example (analysis in the Einstein-Lovelock theory) — which show that classical Einstein gravity may not be applicable to study black hole production in transplanckian collisions. If so, the geometric cross section estimate (4) becomes much less certain, having lost its most serious supporting argument.

The plan of the paper is as follows. In Sec. II we will review the CTS argument. Readers familiar with it may go directly to Sec. III, where we will discuss the danger posed by regions of high curvature and see that curvature becomes large along the transverse plane at the collision moment. This conclusion is reached after the finite width of the shocks due to classical and quantum smearing is taken into account.

The remaining part of the paper deals with the Einstein-Lovelock theory. In Sec. IV we will review the Lovelock Lagrangian and its higher order generalizations. I will also comment on the history of this Lagrangian and its alleged connection to string theory.

In Sec. $\mathrm{V}$ we will write down a metric ansatz for the head-on collision, in a plane-wave approximation. Then we will derive equations of motion, in a small-gradient approximation, in the region of longitudinal light-cone coordinates $|u|,|v| \lesssim 1$. We will also see that the cubic and higher order Lovelock Lagrangians do not contribute in this approximation.

In Sec. VI we will solve the resulting hyperbolic Monge-Ampère equation. First we will see that no usual-sense (smooth) solution exist, even when we consider the smeared out shocks as initial conditions. Then, in the limit of infinitely thin shocks we will find an infinite class of generalized (weak) solutions. We will discuss what this non-uniqueness may mean, and how it can possibly be resolved.

In Sec. VII we will check that the small-gradient approximation made in Sec. V was justified. We will then use the found solutions of the Monge-Ampère equation to write down approximate spacetime metrics. I will explain the differences between the Einstein and Einstein-Lovelock post-collision spacetimes, and their negative implications for the CTS 
argument. I will conclude in Sec. VIII with a summary of the obtained results.

Notation. In the remainder of the paper we work in flat spacetime of $D \geq 5$ dimensions (although we quote some $D=4$ results for comparison). This is legitimate, since the relevant gravitational dynamics happens in a region of $\sim \mathrm{TeV}^{-1}$ size, which is much smaller than the size of extra dimensions. We use Planck units, setting $8 \pi G=1$ in the $D$-dimensional Einstein-Hilbert action. Greek indices $\mu, \nu, \ldots$ run through all $D$ coordinates, Latin $i, j, \ldots$ only through $D-2$ transverse directions. Spacetime signature is $(+,-,-, \ldots)$, and the curvature tensor definitions are $R_{\nu \alpha \beta}^{\mu}=\partial_{\alpha} \Gamma_{\nu \beta}^{\mu}-\ldots$ and $R_{\mu \nu}=R_{\mu \alpha \nu}^{\alpha}$ ('Landau-Lifshitz timelike conventions').

\section{CLOSED TRAPPED SURFACE ARGUMENT}

The basic ingredient in existing discussions of black hole formation in particle collisions is the gravitational field of a fast point particle moving along a straight line. In the limit of infinite boost $\gamma=E / m \rightarrow \infty$ and fixed energy $E$, this field takes the form of a shock wave

$$
d s^{2}=d \bar{u} d \bar{v}-\Phi(\bar{x}) \delta(\bar{u}) d \bar{u}^{2}-d \bar{x}^{2}
$$

Here $\bar{u}=\bar{t}-\bar{z}, \bar{v}=\bar{t}+\bar{z}$, the particle is moving in the positive $\bar{z}$ direction, and $\bar{x}$ denotes the $D-2$ transverse coordinates.

Einstein's equations with the lightlike source

$$
T_{\bar{u} \bar{u}}=E \delta(\bar{u}) \delta^{D-2}(\bar{x})
$$

give the following equation for the shock wave profile $\Phi(\bar{x})$ :

$$
-\frac{1}{2} \nabla^{2} \Phi=E \delta^{D-2}(\bar{x})
$$

Thus we get $^{2}\left(\bar{r}=|\bar{x}| ; \Omega_{D-3}\right.$ is the volume of the unit $D-3$ sphere $)$

$$
\begin{aligned}
\Phi & =-\frac{E}{\pi} \ln \bar{r} \quad(D=4), \\
\Phi & =\frac{2 E}{\Omega_{D-3}(D-4) \bar{r}^{D-4}} \quad(D \geq 5) .
\end{aligned}
$$

\footnotetext{
2 This result can also be derived by boosting the static $D$-dimensional Schwarzschild solution and simultaneously scaling down the rest mass to keep the energy constant. This was the method originally used by Aichelburg and Sexl in $D=4$ [13].
} 
Form (5) of the metric is unsuitable for analyzing the behavior of geodesics crossing the shock at $\bar{u}=0$, which is necessary for understanding the causal structure. For this we must switch to coordinates in which the metric is continuous. This is accomplished by the discontinuous coordinate transformation [14, 15, 8]

$$
\begin{aligned}
\bar{u} & =u \\
\bar{v} & =v+\Phi \theta(u)+\frac{u \theta(u)(\nabla \Phi)^{2}}{4} \\
\bar{x}^{i} & =x^{i}+\frac{u}{2} \nabla_{i} \Phi(x) \theta(u)
\end{aligned}
$$

(where $\theta$ is the Heaviside step function). In the new coordinates the metric becomes

$$
\begin{aligned}
& d s^{2}=d u d v-H_{i k} H_{j k} d x^{i} d x^{j}, \\
& H_{i j}=\delta_{i j}+\frac{1}{2} \nabla_{i} \nabla_{j} \Phi(x) u \theta(u),
\end{aligned}
$$

and both geodesics and their tangents are continuous across the shock. Introducing polar coordinates in the transverse plane, this metric can be written as (see [14] for $D=4$ )

$$
d s^{2}=d u d v-\left[1+\frac{(D-3) E}{\Omega_{D-3} r^{D-2}} u \theta(u)\right]^{2} d r^{2}-\left[1-\frac{E}{\Omega_{D-3} r^{D-2}} u \theta(u)\right]^{2} r^{2} d \Omega^{2} .
$$

To discuss a collision, we add a second fast particle of the same energy moving opposite to the first one in the negative $z$ direction at a transverse distance (impact parameter) $b$. Assuming that the particles pass each other at $u=v=0$, the combined gravitational field outside the wedge $u, v>0$ can be obtained by superposing the metric (13) with its mirror image:

$$
\begin{aligned}
d s^{2} & =d u d v-\left(H_{i k} H_{j k}+\tilde{H}_{i k} \tilde{H}_{j k}-\delta_{i j}\right) d x^{i} d x^{j}, \\
\tilde{H}_{i j} & =\delta_{i j}+\frac{1}{2} \nabla_{i} \nabla_{j} \Phi(x-(b, 0, \ldots, 0)) v \theta(v) .
\end{aligned}
$$

Such superposition is legal, because the excised region $u, v>0$ (wedge $F$ in Fig. (1) is precisely the future light cone of the collision plane of the shocks. By causality, outside this region the shocks will not be able to influence each other.

The metric for $u, v>0$ must be found by solving the characteristic initial value problem for Einstein's equations. This task is complicated by the fact that the shocks after passing each other will focus and develop regions of high curvature. Shock propagation through these regions depends on their detailed structure [14]. 
Nevertheless, we are interested whether this complicated dynamics will lead to black hole formation. Since the complete metric is unknown, we must resort to indirect arguments. The idea [12, 8] is to look for a closed trapped surface (CTS) in the part (16) of the spacetime that we do know. CTS is defined as a closed $(D-2)$-surface whose area decreases locally when propagated along the outer null normals. Equivalently, the outer null normals of such surface have positive convergence [16].

Existence of a CTS in a spacetime solving vacuum Einstein's equations implies presence of a singularity in the future. Assuming Cosmic Censorship, this singularity must be hidden behind a horizon, and we may conclude that a black hole will form. Moreover, the black hole horizon must lie outside the CTS. Using this information, one can get estimates of the horizon area and, via the Area Theorem, of the mass of the formed black hole.

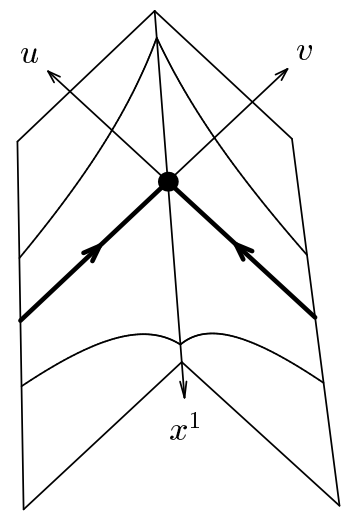

FIG. 2: CTS (19). The trajectories of the colliding particles and the null planes containing the CTS are also shown. All the transverse directions but $x^{1}$ are suppressed.

For the head-on collision $(b=0)$ a CTS is easy to find [12, 8]. It lies in the union of the shock planes $u=0$ and $v=0$ and, when transformed to the $\bar{u}, \bar{v}, \bar{x}$ coordinates, consists of two flat $(D-2)$-dimensional disks of radii

$$
r_{0}=\left(E / \Omega_{D-3}\right)^{1 /(D-3)}
$$

centered at the collision point. In the $u, v, x$ coordinates the CTS is glued out of two halves described by

$$
\begin{aligned}
& u=0, \quad v=\Phi\left(r_{0}\right)-\Phi(r), \quad r \leq r_{0} ; \\
& v=0, \quad u=\Phi\left(r_{0}\right)-\Phi(r), \quad r \leq r_{0} .
\end{aligned}
$$

It is symmetric under rotations of the transverse directions and the reflection $z \rightarrow-z$ (see Fig. 2). Strictly speaking, this surface is a marginal CTS, which means that the outer null 
normals have zero convergence. (Such a surface is also called apparent horizon.) However, moving a small distance inside one can find a true CTS with negative convergence.

For nonzero impact parameters $(b>0)$ the search of trapped surfaces was carried out analytically for $D=4$ by Eardley and Giddings [8] and numerically for $5 \leq D \leq 11$ by Yoshino and Nambu [17]. In every $D$ existence of a marginal CTS was established for impact parameters

$$
b \leq c_{D} R_{\mathrm{Schw}}(E)=c_{D}\left(\frac{2 E}{(D-2) \Omega_{D-2}}\right)^{1 /(D-3)},
$$

where $c_{D} \approx 1.5$ is a numerical constant weakly depending on $D$. This result of classical general relativity if the most serious argument in favor of the geometric cross section for the black hole production rate.

\section{HIGH CURVATURE AT $u=v=0$}

The CTS argument of the previous section was valid for the Einstein gravity with its vacuum equation of motion $R_{\mu \nu}=0$. This equation is used crucially in the Raychaudhuri equation describing how the convergence $\theta$ evolves along the congruence of null normals $\ell^{\mu}$ :

$$
\frac{d \theta}{d \Lambda}=R_{\mu \nu} \ell^{\mu} \ell^{\nu}+\frac{1}{2} \theta^{2}+2(\text { shear })^{2} .
$$

For Ricci-flat spacetimes the first term vanishes. One then concludes that once $\theta$ goes positive, it grows monotonically and becomes infinite at finite affine distance $\Lambda$. This argument is the basis of the proof of singularity theorems 16.

Now suppose that long before the singularity forms, the evolving surface passes through a region of high curvature. If higher order curvature terms are present in the effective gravitational Lagrangian, they will become important and modify the field equations at this point. In general, $R_{\mu \nu}$ will no longer vanish, and one cannot make any conclusions about the dynamics of $\theta$ across the region of high curvature.

From the effective field theory point of view, it seems likely that all interactions allowed by symmetry should become significant in a theory at its natural scale (which is the $D$-dimensional Planck scale in our case). This is the main reason to believe that the gravitational Lagrangian will contain higher curvature terms, with coefficients of natural magnitude $\sim 1$. There are also other hints pointing in the same direction. For instance, 
since the Einstein gravity is non-renormalizable (see, e.g., review [18]), higher curvature counterterms should be included to cancel infinities in loop diagrams. In string theory, which is a finite theory of quantum gravity [19], a particular sequence of higher curvature terms with calculable coefficients appears in the effective action (the $\alpha^{\prime}$-expansion). ${ }^{3}$

It should be noted that singularity theorems of general relativity can also be formulated and proved in presence of matter sources satisfying various positivity assumptions, such as the null energy condition $T_{\mu \nu} \ell^{\mu} \ell^{\nu} \geq 0$. While such conditions are natural for classical matter, they can be violated by the renormalized energy-momentum tensor of quantum fields [21]. So it is unlikely that any useful generalization of singularity theorems exists when quantum effects are taken into account.

These considerations make it clear that appearance of high curvature regions is problematic for the whole CTS argument. Eardley and Giddings tried to address this problem. In particular they noticed that the CTS they constructed lies in the planes of shocks. They proposed to bring it out of these planes by propagating it a small affine distance to the future along the outer null geodesics. This way, they said, one can get a CTS lying everywhere in the region of small curvature. The conclusion was that quantum gravity effects are unlikely to modify the result: the black hole will still form.

I believe that this argument as it stands is incomplete: it misses the fact that curvature may become large on the part of the trapped surface where it crosses the transverse collision plane.

First of all, the only nonzero components of the Riemann tensor of spacetime (15) are

$$
R_{u i u j}=\frac{1}{2} \nabla_{i} \nabla_{j} \Phi(x) \delta(u)
$$

All curvature invariants (contractions of this tensor with itself and the metric) vanish identically. Thus a shock plane by itself is not a region of high curvature. ${ }^{4}$

However, a problematic region does appear when we add a second shock. It is located at

\footnotetext{
${ }^{3}$ For completeness, we have to mention that Loop Quantum Gravity [20] attempts to quantize general relativity non-perturbatively, starting from the pure Einstein-Hilbert action. Since the program is unfinished, and in particular the classical limit is poorly understood, it is hard to say what form the gravitational field dynamics will eventually take. It seems likely that the effective action, as long as this concept is applicable, will have to contain higher curvature terms even in this theory.

${ }^{4}$ This also follows from the fact that the Aichelburg-Sexl wave can be obtained by boosting from manifestly low-curvature regions far away from a static $D$-dimensional black hole.
} 
$u=v=0$, where the shocks collide. At this moment we can form a nonvanishing curvature invariant of the collision spacetime (16):

$$
R_{\mu \nu \lambda \sigma} R^{\mu \nu \lambda \sigma} \propto \sum_{i, j} R_{u i u j} R_{v i v j} \propto \delta(u) \delta(v) .
$$

This equation seems to suggest that curvature becomes large (and even infinite) all along the transverse plane $u=v=0$. However, it would be incorrect to jump to this conclusion too soon. The reason is that infinitely thin shocks are an idealization; in reality the shocks will have a finite width $w$.

There are essentially two reasons for $w>0$. The first, purely classical, reason is that in practice $\gamma=E / m$ is large but not infinite. Because of this, the shocks have width

$$
w_{\text {class }} \sim r / \gamma,
$$

depending on the transverse distance $r$. This width comes out naturally when deriving the shock wave by boosting the static black hole spacetime [22, 14].

The second reason has quantum nature. In relativistic quantum theory coordinates of a particle at rest cannot be measured more precisely than its Compton wavelength $\sim 1 / \mathrm{m}$ (see, e.g., [23]) . For an ultrarelativistic particle, this limit becomes $\sim 1 / E$. Thus the point particle picture used in the CTS argument should not be taken literally. In reality the particles should be thought to have finite size $\sim 1 / E$, which translates to a contribution to the shock width

$$
w_{\text {quan }} \sim 1 / E .
$$

We are interested in curvature at transverse radii $r \sim r_{0}$ relevant for the trapped surface formation and evolution. In practice, for such $r$ we will always have $w_{\text {class }} \ll w_{\text {quan }}$, because the masses of colliding particles are small compared to $1 \mathrm{TeV}$, and thus $\gamma$ is very large. (E.g. $\gamma \sim 10^{6}$ at the LHC, using current-quark masses $m_{u, d} \lesssim 10 \mathrm{MeV}$.) Thus we can estimate

$$
w \sim w_{\text {quan }} \sim 1 / E .
$$

Because of nonzero width, $\delta$-functions in (22), (23) will be smeared out over an interval of length $\Delta u \sim w$. Since the integral has to remain unity, the maximal attained value will be $\sim 1 / w \sim E$. Using this value in (23), we find

$$
\max _{r=r_{0}}\left(R_{\mu \nu \lambda \sigma}\right)^{2}=4(D-2)(D-3) \Omega_{D-3}^{\frac{2}{D-3}} E^{\frac{2(D-4)}{D-3}} .
$$


This formula involves a positive power of $E$, and a large prefactor (increasing from $\sim 300$ to $\sim 670$ for $D=5 \ldots 11)$.

We see that even after finite shock width is taken into account, curvature still becomes large in the region of transverse collision plane relevant for the black hole formation. This means that corrections to the Einstein gravity will become important at this moment. Their subsequent effect on dynamics is hard to predict in general. It is not a priori excluded that this effect will be transient, localized in a small shock-interaction region to the future of $u=v=0$, after which the gravitational field will return to its Einsteinian value. In this case we could push the CTS through this small problematic region, similarly to Eardley and Giddings's proposal, and get to small curvature values where the Einstein gravity would again be applicable. (In the original proposal [8, p. 6] the part of the trapped surface near $u=v=0$ was to be left fixed.) However, I think that such localization is unlikely to happen. It is much more probable that the corrections will be significant up to the values $u, v \sim 1$, and even further. This would mean that the Einstein gravity alone essentially loses its predictive power in the future of the collision plane (a possibility also mentioned by Kancheli [24]).

In the remaining part of the paper I will give an example of how this scenario may be realized, using a particular modification of the Einstein gravity by curvature squared terms.

\section{EINSTEIN-LOVELOCK GRAVITY}

We consider a modification of the $D$-dimensional Einstein gravity by curvature squared terms described by the Lagrangian

$$
\begin{aligned}
\mathcal{L} & =-\frac{1}{2} R \sqrt{-g}+\kappa \mathcal{L}^{(2)}, \\
\mathcal{L}^{(2)} & =\left(R_{\mu \nu \lambda \sigma} R^{\mu \nu \lambda \sigma}-4 R_{\mu \nu} R^{\mu \nu}+R^{2}\right) \sqrt{-g} .
\end{aligned}
$$

The new coupling $\kappa$ is assumed to be $\sim 1$ on the grounds of naturalness. Lagrangian (29) has an interesting property first discovered by Lovelock [11]: it is the only combination of curvature squared terms leading to second order equations of motion for the metric. (A generic combination would produce fourth order equations.)

For this reason theory (28) has been often used in attempts to understand how higher curvature corrections modify the behavior of pure Einstein gravity. Thorough reviews of 
the existing literature can be found in 25] (applications to black holes) and 26] (KaluzaKlein scenarios; braneworlds). Recently, theory (28) was even discussed in connection with black holes produced in particle collisions: the authors of [27] argued that by studying their Hawking evaporation spectra one can measure $\kappa$. However, in this paper we are interested how the Lovelock term manifests itself before rather than after the black hole formation, and whether it may in fact preclude this formation.

Actually, Lovelock 11] has discussed a whole sequence of higher order Lagrangians

$$
\mathcal{L}^{(n)}=\frac{(2 n) !}{2^{n}} R_{\left[\nu_{1} \nu_{2}\right.}^{\nu_{1} \nu_{2}} R_{\nu_{3} \nu_{4}}^{\nu_{3} \nu_{4}} \ldots R_{\left.\nu_{2 n-1} \nu_{2 n}\right]}^{\nu_{2 n-1} \nu_{2 n}} \sqrt{-g}
$$

This gives $R \sqrt{-g}$ for $n=1$ and reduces to (29) for $n=2$.

In general, $\mathcal{L}^{(n)}$ vanishes identically for $D \leq 2 n-1$ and is a total derivative for $D=2 n$ (the generalized Gauss-Bonnet theorem [28]). For $D \geq 2 n+1, \mathcal{L}^{(n)}$ leads to second order equations of motion. The corresponding variations were also found by Lovelock [11]:

$$
\begin{aligned}
& \delta\left(\int \mathcal{L}^{(n)} d^{D} x\right)=\int G_{\lambda \sigma}^{(n)} \delta g^{\lambda \sigma} \sqrt{-g} d^{D} x \\
& G_{\lambda \sigma}^{(n)}=-\frac{(2 n+1) !}{2^{n+1}} g_{\lambda[\sigma} R_{\nu_{1} \nu_{2}}^{\nu_{1} \nu_{2}} \ldots R_{\left.\nu_{2 n-1} \nu_{2 n}\right]}^{\nu_{2 n-1} \nu_{2 n}} .
\end{aligned}
$$

For $n=1$ this coincides with the Einstein tensor.

Explicit expressions for $\mathcal{L}^{(n)}$ and $G_{\lambda \sigma}^{(n)}(n \leq 4)$ with terms of equivalent tensor index structure collected as in (29) can be found in [29]. We won't need them, since in practice for $n \geq 2$ it is much easier to work with (30) and (32).

Sometimes in the literature the theory (29) in $D=5$ is attributed to Cornelius Lanczos, citing his papers [30]. In fact, however, Lanczos never went beyond $D=4 .^{5}$ The name 'Gauss-Bonnet' often associated with gravity theories based on Lagrangians (30) is also an example of unfortunate terminology, since in $D \geq 2 n+1$ these 'dimensionally continued' Gauss-Bonnet densities are no longer associated with topological invariants (which is precisely why they become interesting from the dynamical point of view). In this paper we will use the term Einstein-Lovelock gravity for the theories based on (29), (30).

It is often incorrectly stated that $\mathcal{L}^{(2)}$ is the only combination of curvature squared corrections to the Einstein gravity consistent with string theory. This claim is based on a (correct)

\footnotetext{
${ }^{5}$ In 1932 he did not even include $\left(R_{\mu \nu \lambda \sigma}\right)^{2}$ in the Lagrangian; in 1938 he proved that (29) is a topological invariant density in $D=4$. I would like to thank N. Deruelle, J. Madore and J. Zanelli for the interesting discussions of this historical matter.
} 
result of Zwiebach [31], who showed that (28) is the only curvature squared gravity theory in which the quadratic term in the expansion of the action around flat space is the same as in the pure Einstein gravity. In particular, the graviton is the only particle in the perturbative spectrum, and unphysical ghost poles usually associated with curvature squared terms (see, e.g., [32]) are absent. However, one should remember that only on-shell effective action can be defined in string theory. This is especially clear when this action is computed from the S-matrix, but is also true for the sigma model $\beta$-function method (see, e.g., [33]). Thus there always remains field-redefinition freedom, which can be used for example to change the coefficients in front of or completely remove $\left(R_{\mu \nu}\right)^{2}$ and $R^{2}$ terms in (29). All these actions would be equally consistent with the string S-matrix and, thus, with string theory [34]. In a sense, string theory does not have much to say about off-shell dynamics of quantum gravity.

I thus prefer to think of higher curvature theories like (28) not as fundamental microscopic theories, but as effective field theories of gravity (see, e.g., recent discussion in [35]), with the hope that they may capture some aspects of gravitational field dynamics in presence of regions of high curvature.

\section{METRIC ANSATZ AND EQUATIONS OF MOTION}

We will now study the effect of the Lovelock term (29) on the collision spacetime at $u, v>0$. For simplicity, we will analyze the head-on collision $(b=0)$. In principle, the method could also be used to study the nonzero impact parameter case, most certainly with similar conclusions. Remember that we always assume $D \geq 5$.

Our collisions are transplanckian: $E \gg 1$. It is this situation that can lead to formation of a large classical black hole according to the common lore [7, 8, 4]. For $E \sim 1$ we would be speaking about Planck-size black holes, for which quantum gravity effects are significant without doubt.

Our goal is to find the metric to the future of the collision plane up to $u, v \lesssim 1$, at transverse radii $r \sim r_{0}$. Since $r \gg 1$, we can neglect transverse derivatives of the metric: at $u, v \lesssim 1$ they are suppressed by $1 / r$ compared to the longitudinal ones [see (16), (15)]. Neglecting transverse derivatives means that we approximate the metric near a given point 
$\mathcal{P}$ in the collision plane located at $r \sim r_{0}$ by a plane wave collision metric (see [36])

$$
d s^{2}=e^{L(u, v)} d u d v-[1+A(u, v)]^{2}\left(d y^{1}\right)^{2}-[1+B(u, v)]^{2} \sum_{i=2}^{D-2}\left(d y^{i}\right)^{2}
$$

Here $y^{i}$ are Cartesian coordinates in the transverse plane near $\mathcal{P}$, with the $y^{1}$ axis pointing in the direction of the collision point, and $y^{2}, \ldots y^{D-2}$ in the $D-3$ orthogonal directions (see Fig. 31).

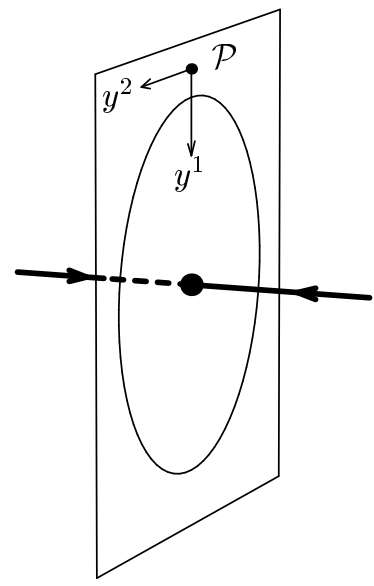

FIG. 3: The transverse collision plane. Shown are the trajectories of the colliding particles and the circular intersection with the CTS (19).

The initial conditions to be imposed on $L, A, B$ outside the future wedge $F(u, v>0)$ are obtained by matching (33) with (16), (15). We find $(u, v \notin F)$

$$
\begin{aligned}
& L(u, v)=0 \\
& A(u, v)=\varepsilon[u \theta(u)+v \theta(v)] \\
& B(u, v)=-\frac{\varepsilon}{D-3}[u \theta(u)+v \theta(v)]
\end{aligned}
$$

where

$$
\varepsilon=\frac{(D-3) E}{\Omega_{D-3} r^{D-2}} \sim E^{-1 /(D-3)} \ll 1 .
$$

To derive the equations of motion, it is convenient to work with a more general metric ${ }^{6}$

$$
d s^{2}=e^{L(u, v)} d u d v-\sum_{i=1}^{D-2}\left[A^{(i)}(u, v)\right]^{2}\left(d y^{i}\right)^{2} .
$$

\footnotetext{
${ }^{6}$ In the rest of this section we suppress Einstein's convention about summing in repeated indices, and indicate all necessary summations explicitly.
} 
The nonzero components of the Riemann tensor are $\left(A_{u}^{(i)}, L_{u v}, \ldots\right.$ are partial derivatives $)$

$$
\begin{aligned}
R_{u v u v} & =\frac{1}{2} e^{L} L_{u v}, \\
R_{u i u i} & =\left[A_{u u}^{(i)}-A_{u}^{(i)} L_{u}\right] A^{(i)}, \\
R_{u i v i} & =A_{u v}^{(i)} A^{(i)} \\
R_{v i v i} & =\left[A_{v v}^{(i)}-A_{v}^{(i)} L_{v}\right] A^{(i)}, \\
R_{i j i j} & =-2 e^{-L}\left[A_{u}^{(i)} A_{v}^{(j)}+A_{v}^{(i)} A_{u}^{(j)}\right] A^{(i)} A^{(j)} .
\end{aligned}
$$

We will now make an a priori assumption that

$$
|\nabla L|,\left|\nabla A^{(i)}\right| \ll 1 \quad(u, v \lesssim 1)
$$

which also implies

$$
L \approx 0, \quad A^{(i)} \approx 1 \quad(u, v \lesssim 1)
$$

Validity of this small-gradient approximation will have to be checked after a solution is found. For now we see that it is compatible with the initial conditions (34).

The approximate Riemann tensor is found by applying (38) and (39) in (37). We have:

$$
\begin{aligned}
R_{u v u v} & \approx \frac{1}{2} L_{u v}, \\
R_{u i u i} & \approx A_{u u}^{(i)}, \\
R_{u i v i} & \approx A_{u v}^{(i)}, \\
R_{v i v i} & \approx A_{v v}^{(i)},
\end{aligned}
$$

all other components being $\approx 0$.

The vacuum equations of the Einstein-Lovelock gravity are

$$
-\frac{1}{2} G_{\lambda \sigma}+\kappa G_{\lambda \sigma}^{(2)}=0
$$

The $u v$ and $i i$ components of this tensor equation will give $D-1$ independent equations for $D-1$ functions $L$ and $A^{(i)}$. The nonzero components of $G_{\lambda \sigma}$ and $G_{\lambda \sigma}^{(2)}$ in the small-gradient 
approximation are easy to find using (40). For $G_{\lambda \sigma}$ we have:

$$
\begin{aligned}
G_{u v} & =\sum_{i} A_{u v}^{(i)}, \\
G_{u u} & =-\sum_{i} A_{u u}^{(i)}, \\
G_{v v} & =-\sum_{i} A_{v v}^{(i)}, \\
G_{i i} & =-2 L_{u v}-4 \sum_{k \neq i} A_{u v}^{(k)} \quad(1 \leq i \leq D-2) .
\end{aligned}
$$

For $G_{\lambda \sigma}^{(2)}$ we use the general expression (32) with $n=2$. Because of the antisymmetrization in the r.h.s. of (32), only terms for which $\sigma, \nu_{1}, \ldots, \nu_{2 n}$ are all different can contribute. Also, looking at (40), we see that $u$ or $v$ must be present in each pair of indices $\left(\nu_{1}, \nu_{2}\right), \ldots$, $\left(\nu_{2 n-1}, \nu_{2 n}\right)$. Using such reasoning, it is easy to see that for $n=2$ the only nonzero components $\operatorname{are}^{7}$

$$
G_{i i}^{(2)}=16 \sum_{\substack{j \neq k \\ j, k \neq i}}\left[A_{u v}^{(j)} A_{u v}^{(k)}-A_{u u}^{(j)} A_{v v}^{(k)}\right] \quad(1 \leq i \leq D-2) .
$$

We now go back to our original ansatz (33), putting

$$
A^{(1)}=1+A(u, v), \quad A^{(i)}=1+B(u, v) \quad(2 \leq i \leq D-2) .
$$

Substituting (42) and (43) in (41), we have three independent equations for $L, A, B$. The simplest is the $u v$-equation, to which only the Einstein tensor contributes:

$$
A_{u v}+(D-3) B_{u v}=0
$$

This equation is easy to solve. Namely, it implies that the relation

$$
B(u, v)=-\frac{A(u, v)}{D-3}
$$

satisfied by the initial data (34), will continue to hold for $u, v>0$.

There remain two $i$ equations $(i=1,2)$. After $B$ is excluded using (46), they can be brought to the form

$$
\begin{aligned}
& \kappa\left[A_{u u} A_{v v}-\left(A_{u v}\right)^{2}\right]+\frac{D-3}{16(D-4)} A_{u v}=0 \\
& L_{u v}=A_{u v}
\end{aligned}
$$

\footnotetext{
${ }^{7}$ Notice that for $n \geq 3$ the given conditions on indices are incompatible. This means that the higher order Lovelock tensors vanish in the small-gradient approximation.
} 
After a function $A(u, v)$ satisfying (47) is found, it will be easy to find $L(u, v)$ from (48). Taking the initial conditions (34) into account, we will have

$$
L(u, v)=A(u, v)-\varepsilon[u \theta(u)+v \theta(v)] .
$$

Thus we reduced the problem to solving a single nonlinear second order PDE (47). In the pure Einstein theory $(\kappa=0)$ the nonlinearity disappears, and we see that the solution for $u, v>0$ is given by the same formulas (34) as the initial data. For $\kappa \sim 1$ this is no longer a valid solution, since it has

$$
A_{u u} A_{v v}-A_{u v}^{2}=\varepsilon^{2} \delta(u) \delta(v),
$$

which becomes large at $u=v=0$ (namely, $\sim \varepsilon^{2} E^{2} \gg 1$, when the finite shock width $w \sim 1 / E$ and the resulting smearing of the $\delta$-functions are taken into account).

PDE (477) belongs to the well-known family of hyperbolic Monge-Ampère equations. It may be noticed (see [37]) that it can be derived from the action

$$
\begin{aligned}
S_{\mathrm{MA}} & =\int d u d v\left\{\frac{\kappa}{3}\left(A_{u}^{2} A_{v v}-2 A_{v} A_{u} A_{u v}+A_{v}^{2} A_{u u}\right)+\frac{D-3}{16(D-4)} A_{u} A_{v}\right\} \\
& =\int d t d z\left\{\frac{\kappa}{3} \epsilon^{a a^{\prime}} \epsilon^{b b^{\prime}} \partial_{a} A \partial_{b} A \partial_{a^{\prime} b^{\prime}} A+\frac{D-3}{64(D-4)} \eta^{a b} \partial_{a} A \partial_{b} A\right\}
\end{aligned}
$$

which we should consider as a 2-dimensional truncation of the full Einstein-Lovelock action relevant for our problem. The first term in this action uses only the totally antisymmetric tensor to form contractions. For this reason the corresponding nonlinear term in Eq. (47) is invariant with respect to Euclidean rotations as well as Lorentz transformations. The linear wave equation term in (47) is of course only Lorentz-invariant.

\section{SOLVING THE MONGE-AMPÈRE EQUATION}

\section{A. Smooth solutions}

Hyperbolic Monge-Ampère equations have exceptional character among the nonlinear second order hyperbolic PDEs: they can be reduced to a system of five first order characteristic ODEs, while in general (in two dimensions) one needs eight [38, p. 495]. However, in case of Eq. (47), because it has constant coefficients, this general theory is superseded by an even simpler method based on the Legendre transform. 
Let us rewrite (47) as

$$
A_{u u} A_{v v}-A_{u v}^{2}+\lambda A_{u v}=0, \quad \lambda=\frac{D-3}{16(D-4) \kappa},
$$

substitute

$$
\begin{aligned}
& A=\lambda(\tilde{A}+u v), \\
& \tilde{A}_{u u} \tilde{A}_{v v}-\tilde{A}_{u v}^{2}-\tilde{A}_{u v}=0,
\end{aligned}
$$

and apply the Legendre transform [38, p. 32]. This results in a linear Poisson equation, which is readily solved. In this way Ignatov and Poponin [39] obtained an exact solution of the original Monge-Ampère equation (52) in an implicit form, depending on two arbitrary functions $F(\xi)$ and $G(\eta)$ :

$$
A(u, v)=\lambda\left[F(\xi)+G(\eta)+F^{\prime}(\xi) G^{\prime}(\eta)\right],
$$

where $\xi$ and $\eta$ must be found from

$$
\begin{aligned}
& u=\eta+F^{\prime}(\xi), \\
& v=\xi+G^{\prime}(\eta) .
\end{aligned}
$$

For vanishing at infinity small functions $F, G$ this solution describes interaction of two colliding pulses of the $A$ field. The pulses emerge from the interaction region with their shape unchanged, resuming the motion along the pre-collision world lines.

In general, solution (55) is applicable if the transformation $(\eta, \xi) \rightarrow(u, v)$ given by (56) is one-to-one. This means that its Jacobian should not vanish:

$$
\frac{\partial(u, v)}{\partial(\eta, \xi)}=1-F^{\prime \prime}(\xi) G^{\prime \prime}(\eta) \neq 0 .
$$

Unfortunately, this condition is violated in our problem. Indeed, to satisfy the initial conditions (34), we would have to take

$$
F(\xi)=G(\xi)=\varepsilon \xi \theta(\xi) .
$$

Because of finite shock width $w \sim 1 / E$ these initial data have to be smeared out on this scale. Still this results in $F^{\prime \prime}(\xi)$ near $\xi=0$ of the order $\sim \varepsilon E \gg 1$. So the Jacobian (57), being equal to unity away from the origin, changes sign and becomes negative near 
$\xi=\eta=0$. The transformation (56) is thus not one-to-one: there is a region of the $u, v$ plane which is covered 3 times, and where the inverse transformation is multiple-valued.

This analysis shows that for initial data (34), even after smearing them out, the exact solution (55) in any case cannot be used verbatim. A region of spacetime appears where this solution is triple-valued, and we have to somehow choose between the three branches. Actually, it turns out that no satisfactory choice is possible. More precisely, any choice introduces discontinuities either in the function $A$ itself (which is totally unacceptable) or in its first derivatives (which brings back the problems which we temporarily resolved by using smoothed out initial data). All of them seem to violate Eq. (52).

To get some insight about the source of these difficulties, I also studied the problem numerically. The conclusion of this study (which I am not going to report here in detail) is that the solution develops singularities characterized by discontinuous first partial derivatives. Evolution beyond these singularities apparently cannot be described by formula (55). A different strategy is required, which I will now proceed to describe.

\section{B. Generalized solutions}

To find a solution, we will have to extend the class of admissible functions $A$, allowing continuous functions with discontinuous first derivatives. [Notice that the initial data (34) corresponding to infinitely thin shocks belong to precisely this class.] Such generalized solutions, called weak in the theory of PDEs 38 , p. 418, 486], are usually defined via integration by parts. In our case weak solutions can be defined, because the Monge-Ampère operator can be written as a divergence

$$
A_{u u} A_{v v}-A_{u v}^{2}=\partial_{v}\left(A_{v} A_{u u}\right)-\partial_{u}\left(A_{v} A_{u v}\right) .
$$

Thus Eq. (52) is equivalent to the conservation law

$$
\partial_{u} J_{v}+\partial_{v} J_{u}=0
$$

where the current $J_{a}$ has components

$$
\begin{aligned}
& J_{v}=-A_{v} A_{u v}+\frac{\lambda}{2} A_{v}, \\
& J_{u}=A_{v} A_{u u}+\frac{\lambda}{2} A_{u} .
\end{aligned}
$$


For smooth functions $A$ the differential form (60) of the conservation law is equivalent to the integral form:

$$
\oint_{\mathcal{C}} \epsilon^{a b} J_{a} d x_{b}=0
$$

for any closed curve $\mathcal{C}$. However, the latter condition does not involve products of second derivatives of $A$ and can be unambiguously checked even when the r.h.s. of (52) cannot be defined. I thus take (63) as the definition of weak solutions of Eq. (152).

In practice, it is always sufficient to check (63) only for infinitesimally small contours $\mathcal{C}$, and this only near special, most dangerous, points. In the rest of the $u, v$ plane the differential equation (52) can still be used. Provided that the first partial derivatives of $A$ are bounded, the nonsingular terms proportional to $\lambda$ drop out of (63) in the limit of small contours. The remaining rule for catching possible $\delta(u) \delta(v)$-type singularities as in (50) takes the form

$$
\int_{\sqrt{u^{2}+v^{2}} \leq \epsilon}\left(A_{u u} A_{v v}-A_{u v}^{2}\right) d u d v=\oint_{\sqrt{u^{2}+v^{2}}=\epsilon} A_{v} d A_{u} .
$$

[This formula has in fact general validity and follows directly from (59).] If there is a $\delta$ function at the origin present in the integrand on the 1.h.s., we will be able to detect it by studying the limit of the r.h.s. as $\epsilon \rightarrow 0$. The latter procedure does not involve products of distributions and is unambiguous.

For further discussion it is convenient to simplify (52) by making the substitution

$$
\begin{aligned}
& A=\mathcal{A}(u, v)+\frac{\lambda}{2} u v, \\
& \mathcal{A}_{u u} \mathcal{A}_{v v}-\mathcal{A}_{u v}^{2}+\lambda^{2} / 4=0 .
\end{aligned}
$$

This PDE is hyperbolic due to positivity of $\lambda^{2} / 4$.

We will no longer attempt to use smeared out initial data, and instead will try to find a solution directly in the limit of infinitely thin shocks. Since in this limit $u=v=0$ is likely to be the most singular point, we change to polar coordinates

$$
u=\rho \sin \varphi, \quad v=\rho \cos \varphi
$$

PDE (66]) becomes

$$
\frac{1}{\rho^{2}}\left[\mathcal{A}_{\rho \rho}\left(\mathcal{A}_{\varphi \varphi}+\rho \mathcal{A}_{\rho}\right)-\left(\mathcal{A}_{\rho \varphi}-\mathcal{A}_{\varphi} / \rho\right)^{2}\right]+\lambda^{2} / 4=0
$$


The crucial fact (easy to guess if one remembers the well-known connection between the homogeneous Monge-Ampère equation and developable surfaces) is that for functions $\mathcal{A}$ of the form $\rho f(\varphi)$ the first most singular term in (68) vanishes. To satisfy the full equation, we have to include in $\mathcal{A}$ terms of order $\rho^{2}$. Thus we are led to the ansatz ${ }^{8}$ :

$$
\mathcal{A}=\rho f(\varphi)+\rho^{2} g(\varphi)
$$

Compatibility with the initial conditions (34) requires:

$$
\begin{aligned}
& f(\varphi)= \begin{cases}\varepsilon \sin \varphi, & \varphi \in[\pi / 2, \pi], \\
0, & \varphi \in[\pi, 3 \pi / 2], \\
\varepsilon \cos \varphi, & \varphi \in[3 \pi / 2,2 \pi],\end{cases} \\
& g(\varphi)=-\frac{\lambda}{4} \sin 2 \varphi,
\end{aligned}
$$

For $\varphi \in[0, \pi / 2]$ the functions $f, g$ have to be found so that PDE (66) is satisfied (see Fig. 4).

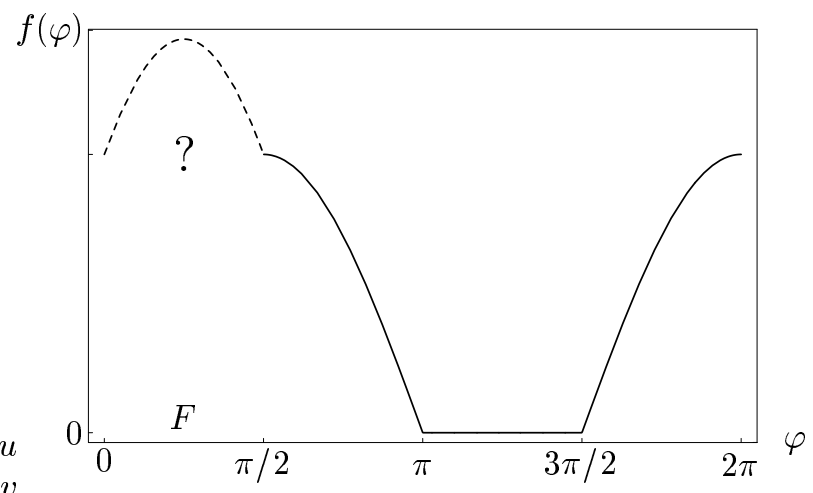

FIG. 4: The initial data for $f(\varphi)$. In the future wedge $F$ (see Fig. 1) the function has to be determined (?). The dashed line denotes the Einsteinian solution (173).

Checking PDE (66) at $u=v=0$ calls for the use of identity (64). An easy calculation shows that

$$
\oint_{\rho=\epsilon} \mathcal{A}_{v} d \mathcal{A}_{u}=\frac{1}{2} \int_{0}^{2 \pi}\left(f^{2}-f^{\prime 2}\right) d \varphi+O(\epsilon)
$$

\footnotetext{
${ }^{8}$ It is possible to consider a more general ansatz, adding terms $\rho^{n} h_{n}(\varphi), n \geq 3$. The functions $h_{n}$ would then be restricted by an infinite sequence of nonlinear ODEs, obtained similarly to (74) and (75) below. These higher order terms can be neglected compared to [69) in the limit of small $\rho$, and so we put them to zero for simplicity. In principle, however, the possibility of them being nonzero further aggravates the non-uniqueness issue to be discussed in Sec. VI.C below.
} 
Taking (70) into account, we get a condition for the absence of $\delta(u) \delta(v)$ in (66):

$$
\int_{0}^{\pi / 2}\left(f^{\prime 2}-f^{2}\right) d \varphi=0 .
$$

As an example when $\delta(u) \delta(v)$ does appear, we can take the Einsteinian solution $\mathcal{A}=$ $\varepsilon[u \theta(u)+v \theta(v)]$, which would correspond to

$$
f(\varphi)=\varepsilon(\sin \varphi+\cos \varphi), \quad \varphi \in[0, \pi / 2] .
$$

And indeed we readily see that (72) is violated.

At $\rho>0$ the solution can be understood in the usual sense, and the use of (64) is unnecessary. We simply plug the ansatz (69) in Eq. (68). Equating the coefficients before $\rho^{-1}$ and $\rho^{0}$ to zero, we get two ODEs:

$$
\begin{aligned}
& \left(f^{\prime \prime}+f\right) g=0, \\
& 2 g g^{\prime \prime}-g^{2}+4 g^{2}+\lambda^{2} / 4=0
\end{aligned}
$$

which have to be solved with the following from (70) boundary conditions

$$
\begin{aligned}
& f(0)=f(\pi / 2)=\varepsilon, \\
& g(0)=g(\pi / 2)=0 .
\end{aligned}
$$

At the first glance we have a problem here, since ODE (174) implies that either $g=0$ or

$$
f^{\prime \prime}+f=0
$$

The first opportunity seems to be excluded by (75), while the only solution of (78) with the boundary conditions (76) is (73), which does not satisfy the no-delta condition (72).

The way out of this impasse is to notice that although $g$ is indeed not allowed to be zero on an interval, it can still vanish at isolated points inside $(0, \pi / 2)$. At these points the derivative $f^{\prime}(\varphi)$ can have jump discontinuities. If on the rest of the interval (78) holds, ODE (74) will still be satisfied.

The general solution of ODE (175) depends on two constants $C, \bar{\varphi}$ and has the form

$$
g(\varphi)= \pm \frac{\lambda}{4}\left\{\sqrt{C^{2}+1} \cos [2(\varphi-\bar{\varphi})]-C\right\}
$$

At points $\varphi_{*}$ where $g\left(\varphi_{*}\right)=0$ we have, in agreement with (75),

$$
g^{\prime}\left(\varphi_{*}\right)= \pm \lambda / 2
$$


The constants $C, \bar{\varphi}$ and the overall sign in (79) can be chosen independently on the two sides of $\varphi_{*}$, provided that both choices are compatible with $g\left(\varphi_{*}\right)=0$. In particular $g^{\prime}\left(\varphi_{*}\right)$ is allowed to flip sign at $\varphi_{*}$. Being multiplied by vanishing $g$, the arising $\delta$-function in $g^{\prime \prime}$ does not violate (75).

Taking all these observations into account, we can write down the following class of solutions to the boundary value problem (74)-(77):

- Functions $f, g$ are continuous on $[0, \pi / 2]$.

- The $f$ satisfies (78) everywhere on $[0, \pi / 2]$ except at some chosen points

$$
0<\varphi_{1}<\ldots<\varphi_{N}<\pi / 2
$$

where $f^{\prime}(\varphi)$ will have jump discontinuities.

- The $g$ vanishes at $\varphi_{1}, \ldots, \varphi_{N}$, and is given by (79) on each subinterval $\left[\varphi_{\alpha}, \varphi_{\alpha+1}\right]$ $\left(\varphi_{0}=0, \varphi_{N+1}=\pi / 2\right.$ are the end points) with

$$
\begin{aligned}
\bar{\varphi} & =\frac{1}{2}\left(\varphi_{\alpha+1}+\varphi_{\alpha}\right), \\
C & =\cot \left(\varphi_{\alpha+1}-\varphi_{\alpha}\right),
\end{aligned}
$$

and an arbitrary overall sign \pm , which can be chosen independently on each subinterval.

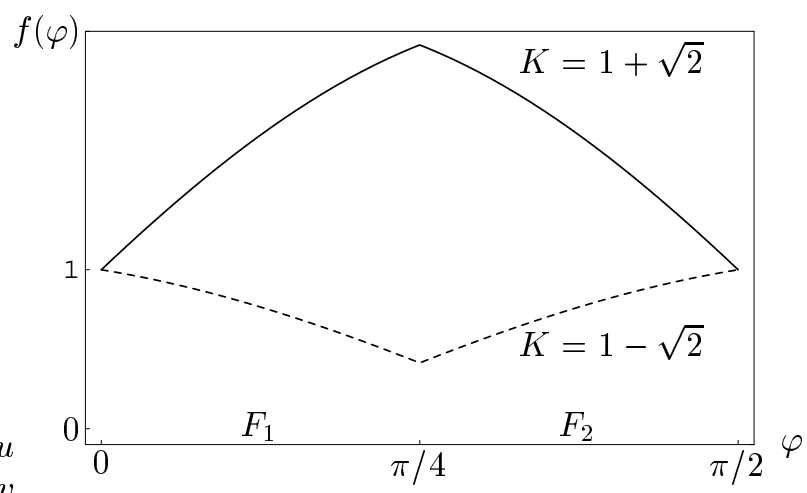

FIG. 5: Solution (84). The future wedge $F$ is split into two equal sectors $F_{1,2}$ (see Fig. (1). The leading behavior of $A(u, v)$ in these sectors is given by $u+K v$ and $v+K u$.

Within this class it becomes possible to satisfy the no-delta condition (72). The simplest and most symmetric example can be constructed using one intermediate point $\varphi_{1}=\pi / 4$ 
(see Fig. 5). From continuity and (78) we must have

$$
f(\varphi)=\varepsilon \times \begin{cases}\cos \varphi+K \sin \varphi, & \varphi \in[0, \pi / 4] \\ \sin \varphi+K \cos \varphi, & \varphi \in[\pi / 4, \pi / 2] .\end{cases}
$$

The constant $K$ is determined from (72). We find that two values are allowed:

$$
K=1 \pm \sqrt{2}
$$

For $g(\varphi)$ we have:

$$
g(\varphi)= \pm \frac{\lambda}{4} \times \begin{cases}\sqrt{2} \cos (2 \varphi-\pi / 4)-1, & \varphi \in[0, \pi / 4] \\ \sqrt{2} \cos (2 \varphi-3 \pi / 4)-1, & \varphi \in[\pi / 4, \pi / 2] .\end{cases}
$$

Near the point $u=v=0$ the leading $\rho f(\varphi)$ behavior of these solutions (as of all the solutions in the class described above) is piecewise linear in terms of the $u, v$ coordinates, with the $\rho^{2} g(\varphi)$ term being a small correction (see Fig. [).

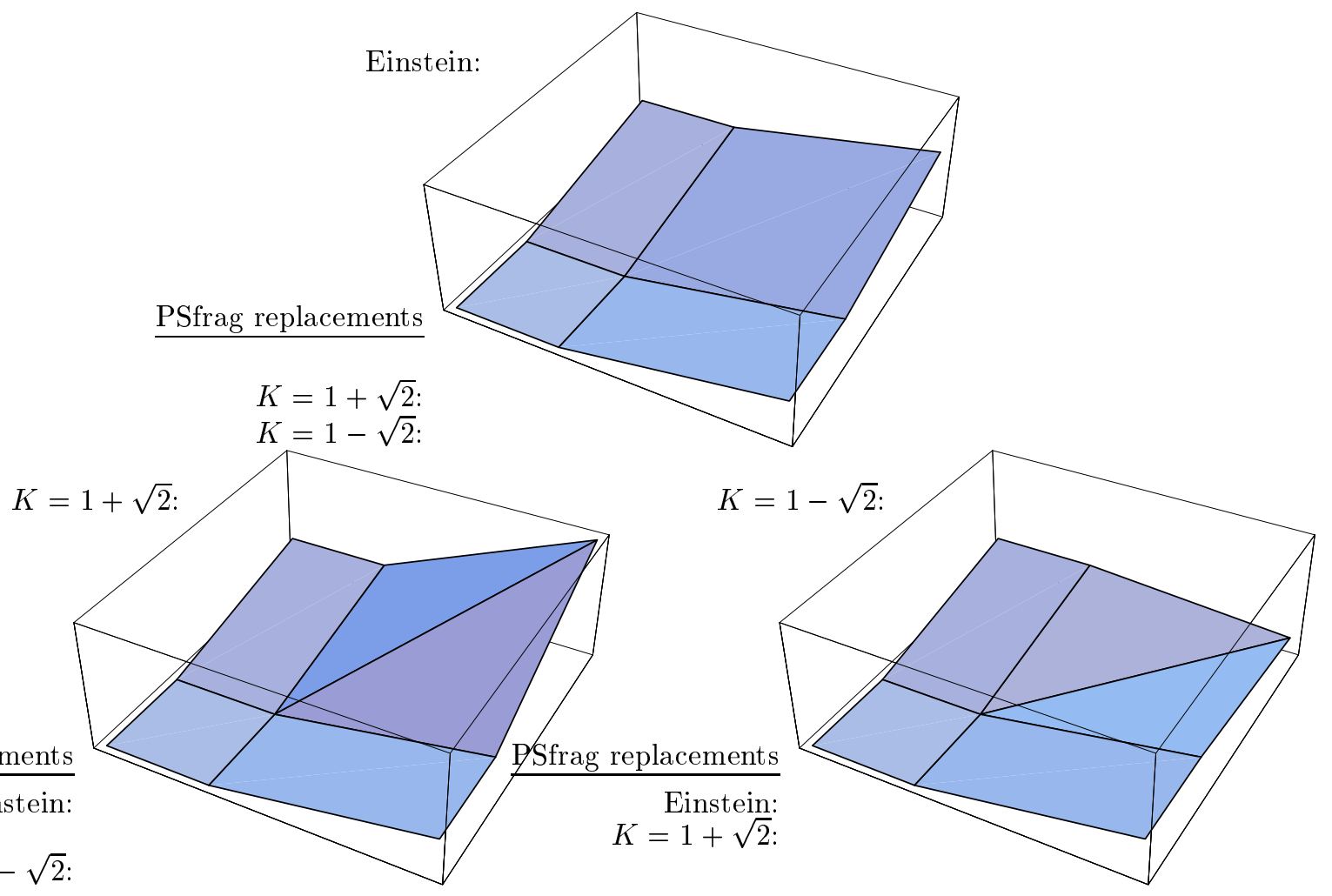

FIG. 6: The leading behavior of $A(u, v)$ near $u=v=0$ for the pure Einstein case (73) and for the Einstein-Lovelock solution (84). The time flow is from the left to the right. 
A more general example, which we will need in a discussion below, utilizes two symmetric intermediate points $\varphi_{1}=\phi, \varphi_{2}=\pi / 2-\phi$, where $\phi \in[0, \pi / 4]$. The function $f$ is given by

$$
f(\varphi)=\varepsilon \times \begin{cases}\cos \varphi+K \sin \varphi, & \varphi \in[0, \phi], \\ \frac{\cos \phi+K \sin \phi}{\cos \phi+\sin \phi}(\cos \varphi+\sin \varphi), & \varphi \in[\phi, \pi / 2-\phi], \\ \sin \varphi+K \cos \varphi, & \varphi \in[\pi / 2-\phi, \pi / 2],\end{cases}
$$

where the $K$ is fixed by (72) to be

$$
K=1 \pm \sqrt{1+\cot \phi}
$$

The allowed functions $g$ can be written down according to the rules given above. For $\phi=\pi / 4$ this example reduces to the previous one.

Taking even more general configurations of intermediate points and imposing the nodelta condition (72), we can get infinitely many solutions of PDE (66) having the form of the ansatz (69) and satisfying the initial conditions (701). Solutions of the original MongeAmpère equation (52) are then given by Eq. (65).

\section{Discussion of non-uniqueness}

Let us repeat the logic of the preceding discussion. In Sec. VI.A we convinced ourselves that solutions of the Monge-Ampère equation (52) with the smeared out initial conditions (34) run into singularities, characterized by discontinuous first partial derivatives. The very meaning of a solution in presence of such discontinuities needs to be reconsidered. For this reason in Sec. VI.B we introduced the definition of weak solutions, using a standard procedure of integration by parts. ${ }^{9}$ Working in the limit of infinitely thin shocks, we found a whole class of weak solutions with the required initial conditions. It is of course satisfying to find that solutions according to the new definition exist, but how should we interpret their non-uniqueness?

In my opinion, there are two alternative possibilities. First, it is possible that this nonuniqueness can be resolved by performing a detailed study of PDE (152) with smeared out

\footnotetext{
${ }^{9}$ Notice that for smooth functions the concepts of usual and weak solutions are equivalent. It is only in presence of discontinuous first derivatives that the usual notion is not applicable, and we have to use the new definition.
} 
initial data at small but finite shock width $w \sim 1 / E$. As we have just discussed, the singularities will still appear. But perhaps by studying their approach one may discover a preferred way to continue the solution past them. This would require techniques beyond what we use in this paper. However, we would like to point out that even if a 'true' unique solution is found in this way, it will still have to satisfy our weak solution criteria from Sec. VI.B. Because of this it seems likely (although cannot be guaranteed) that this 'true' solution will be close to one of the weak solutions we found in Sec. VI.B.

The second, more intriguing, possibility is that the encountered non-uniqueness is fundamental. Then its interpretation can be most naturally given in quantum theory. It would simply mean that beyond the shock collision the gravitational field wavefunction ceases to be concentrated on a single classical configuration. Instead, it spreads out over all allowed solutions of the equations of motion. In this case we would have to sum over all solutions, using $\exp (i S)$, where $S$ is the on-shell value of the action, as the weight.

It should be noted that the problem of non-uniqueness of weak solutions is well known in the theory of PDEs. It is present for instance in the theory of first order quasilinear hyperbolic systems, where the weak solutions in question are shock waves. There the nonuniqueness is resolved by imposing the physically motivated 'entropy condition' 40].

One can try to do something similar in spirit for our PDE (52). For example, one may try to identify higher order currents that are conserved for usual smooth solutions, and impose their conservation as an additional constraint on weak solutions. In fact, the starting point of our discussion of weak solutions was to rewrite the Monge-Ampère equation (52) itself as the current conservation condition (60). Going a step further, let us introduce a symmetric 2-tensor $T_{a b}$ with components

$$
\begin{aligned}
& T_{u u}=(\nabla A)^{2} A_{u u}, \\
& T_{v v}=(\nabla A)^{2} A_{v v}, \\
& T_{u v}=T_{v u}=-(\nabla A)^{2} A_{u v}
\end{aligned}
$$

We have

$$
\begin{aligned}
& \partial_{v} T_{u u}+\partial_{u} T_{v u}=2 A_{v}\left(A_{u u} A_{v v}-A_{u v}^{2}\right), \\
& \partial_{v} T_{u v}+\partial_{u} T_{v v}=-2 A_{u}\left(A_{u u} A_{v v}-A_{u v}^{2}\right) .
\end{aligned}
$$

Actually, the appearance of the Monge-Ampère operator in the r.h.s. is not surprising: $T_{a b}$ is nothing but the energy-momentum (EM) tensor of the homogeneous Monge-Ampère 
equation, Eq. (52) with $\lambda=0$. This equation corresponds to the first term in the action (51). To define the symmetric EM tensor, we covariantize this term:

$$
\int d^{2} x \epsilon^{a a^{\prime}} \epsilon^{b b^{\prime}} \partial_{a} A \partial_{b} A \partial_{a^{\prime} b^{\prime}} A \longrightarrow \int d^{2} x \sqrt{g} E^{a a^{\prime}} E^{b b^{\prime}} \partial_{a} A \partial_{b} A \nabla_{a^{\prime}} \partial_{b^{\prime}} A
$$

and differentiate with respect to the auxiliary 2-dimensional metric $g_{a b}$. The result of this computation is (89) (up to a factor of $3 / 2$ ).

The EM tensor of the full equation (52) differs from (89) by a trivial harmless piece associated with the quadratic term $(\partial A)^{2}$ in (51). In imposing the EM conservation at the singular point $u=v=0$ that piece is irrelevant. Transforming the conservation laws (90), (91) to their integral form, we conclude that the EM tensor will be conserved if

$$
\oint_{\rho=\epsilon}(\nabla A)^{2} d A_{u}, \quad \oint_{\rho=\epsilon}(\nabla A)^{2} d A_{v} \rightarrow 0 \quad(\epsilon \rightarrow 0) .
$$

Applied to the ansatz (69), this gives two conditions on $f(\varphi)$, conveniently written down as one complex condition:

$$
\oint d \varphi e^{i \varphi}\left(f^{\prime 2}+f^{2}\right) \cdot\left(f+f^{\prime \prime}\right)=0 .
$$

When this condition is imposed, the class of weak solutions from Sec. VI.B is reduced, but not eliminated. The simplest solution (84) does not satisfy (94) and would not be allowed. However, already among the one-parameter family (87) there is a solution [corresponding to the numerical value $\phi \approx 0.322$ and the minus sign in (88)] which passes the new criterion. Introducing more intermediate points, we will still have an infinitude of solutions satisfying both the no-delta condition (72) and the EM conservation (94).

It seems likely that a whole hierarchy of currents conserved on the smooth solutions of PDE (52) can be found. In fact, it is possible [41] to map the Monge-Ampère equation (66) to the Born-Infeld equation

$$
\left(1+\Phi_{x}^{2}\right) \Phi_{t t}-2 \Phi_{t} \Phi_{x} \Phi_{t x}-\left(1-\Phi_{t}^{2}\right) \Phi_{x x}=0
$$

or to the first order system describing dynamics of the Chaplygin gas

$$
\left\{\begin{array}{l}
P_{t}=P P_{x}+Q^{-3} Q_{x}, \\
Q_{t}=(P Q)_{x} .
\end{array}\right.
$$

Both (95) and (96) are known to possess infinitely many conserved quantities [42]. Whether translating them back to the Monge-Ampère variables and imposing on the weak solutions is physically motivated, or can help resolve the non-uniqueness, is an open question. 
This is as far as our explorations have taken us in the attempts to resolve the issue of non-uniqueness. Unfortunately, the problem still remains. In particular, at present we are unable to decide which one of the two possibilities outlined at the beginning of this subsection is realized. However, both of them allow for comparison with the pure Einstein case, to which we now proceed.

\section{APPROXIMATE POST-COLLISION METRICS - COMPARISON}

In Sec. V, when deriving the equations of motion, we introduced a small-gradient assumption (38), which for our particular ansatz (33) takes the form

$$
|\nabla A|,|\nabla B|,|\nabla L| \ll 1 \quad(u, v \lesssim 1) .
$$

This was compatible with the initial data, but could not be taken for granted for the full solution. So before we use the solutions found in Sec. VI.B, we have to check (97).

The functions $B$ and $L$ are trivially related to $A$ by (46) and (49). Thus it suffices to check (97) only for $A(u, v)$, which is given by (65) and (69). To estimate the gradient of (69), we compute

$$
\begin{aligned}
\nabla_{u}[\rho f(\varphi)] & =f(\varphi) \sin \varphi+f^{\prime}(\phi) \cos \varphi, \\
\nabla_{u}\left[\rho^{2} g(\varphi)\right] & =\rho\left[2 g(\varphi) \sin \varphi+g^{\prime}(\varphi) \cos \varphi\right]
\end{aligned}
$$

Analogous formulas with $\sin \varphi$ and $\cos \varphi$ interchanged are valid for $\nabla_{v}$. Since $f(\varphi)$ will typically be of order $\varepsilon \ll 1$ [as in (84) and [87)], the $\rho f(\varphi)$ term passes the small-gradient check. Further, the size of $g(\varphi)$ is set by the parameter $\lambda$, which will be $\sim 1$ for $\kappa \sim 1$. It follows that the $\rho^{2} g(\varphi)$ term, as well as the term $\lambda u v / 2$ in (65) , will also pass the check, provided that we restrict $u, v$ to a somewhat smaller region

$$
u, v \lesssim \rho_{\max } \ll 1,
$$

where the precise value of $\rho_{\max }$ has to be chosen depending on the required accuracy. This value is insensitive to $E$, which determines the shock width $w \sim 1 / E$. This means that the found solutions do take us out of the shock interaction region before the small-gradient approximation breaks down.

The plane wave collision metrics (33) corresponding to the solutions of Sec. VI.B approximate the spacetime near the chosen point $\mathcal{P}$ in the transverse plane. They implicitly depend 
on $\mathcal{P}$ through the parameter $\varepsilon$. To assure the consistency of neglecting transverse derivatives, we have to assume that all other free parameters, such as the choice of intermediate points $\varphi_{\alpha}$, depend weakly on or are independent of $\mathcal{P}$. Then we can patch the solutions for different $\mathcal{P}$ 's together. The resulting approximate metric has the form

$$
d s^{2}=e^{L(u, v, r)} d u d v-[1+A(u, v, r)]^{2} d r^{2}-[1+B(u, v, r)]^{2} d \Omega^{2},
$$

where the dependence of $L, A, B$ on the transverse radius is now made explicit. These are the metrics described in the Introduction. The final range of their validity is

$$
r \sim r_{0}, \quad u, v \lesssim \rho_{\max }
$$

In the pure Einstein case, the approximate post-collision metric at $r \sim r_{0}, u, v \lesssim 1$ would have the same form (101), except that the function $A$ has to be found from the linear wave equation $A_{u v}=0$ (see Sec. V). Thus we have

$$
\begin{aligned}
& A_{\mathrm{E}}(u, v, r)=\varepsilon(r) \cdot[u \theta(u)+v \theta(v)], \\
& B_{\mathrm{E}}(u, v, r)=-\frac{A_{\mathrm{E}}(u, v, r)}{D-3}, \\
& L_{\mathrm{E}}(u, v, r)=0 .
\end{aligned}
$$

Comparing the pure Einstein and the Einstein-Lovelock spacetimes, we see that they are quite different. In the Einstein case the Riemann tensor is concentrated on the null planes, while in the Einstein-Lovelock case it has additional $\delta$-function singularities of comparable strength on the planes $u / v=\tan \varphi_{\alpha}$ corresponding to the intermediate points (81). This difference persists as far as we can trace the solutions, that is up to $u, v \sim \rho_{\max }$.

It is instructive to see what happens with the dynamics of CTS in such a spacetime. In Einstein gravity, once a CTS appeared, it can be evolved into the future along its null normals. The CTS property is preserved by such evolution because of the Raychaudhuri equation (21). This fact crucially depends on the inequality

$$
R_{\mu \nu} \ell^{\mu} \ell^{\nu} \geq 0
$$

(for any lightlike $\ell^{\mu}$ ), which follows from Einstein's equations when the matter sources are either absent or satisfy appropriate positivity conditions [16]. 
The Ricci tensor of spacetime (101) is best written down in the same local coordinates $u, v, y^{i}$ that we used in Eq. (33). Neglecting transverse derivatives, and using the smallgradient approximation, we have

$$
\begin{aligned}
R_{u v} & \approx-L_{u v}=-A_{u v}, \\
R_{y^{1} y^{1}} & \approx 4 A_{u v}, \\
R_{y^{i} y^{i}} & \approx 4 B_{u v}=-\frac{4 A_{u v}}{D-3} \quad(2 \leq i \leq D-2)
\end{aligned}
$$

[where we used (46) and (49) to express $B$ and $L$ via $A]$. We see that in the EinsteinLovelock case $R_{\mu \nu} \neq 0$ in the future wedge. Most notably, $R_{\mu \nu}$ will have singularities on the planes $u / v=\tan \varphi_{\alpha}$, with the leading behavior $\propto \rho \delta(\varphi)$. The magnitudes and signs of these singularities depend on the particular choice of solution.

To see what happens with condition (106), let us pick a light-like vector $\ell^{\mu}=\left(\ell^{u}, \ell^{v}, \ell^{i}\right)$. Since the metric components do not differ much from their Minkowskian values (only their second derivatives do), we have

$$
\ell^{u} \ell^{v}-\left(\ell^{i}\right)^{2} \approx 0
$$

Using (107) and (108), it is easy to compute

$$
R_{\mu \nu} \ell^{\mu} \ell^{\nu}=2 A_{u v}\left[\left(\ell^{1}\right)^{2}-\frac{D-1}{D-3} \sum_{i=2}^{D-2}\left(\ell^{i}\right)^{2}\right]
$$

The signs of both factors in this expression are undetermined, and so Ricci tensor (107) will generally violate condition (106).

We thus see that in the considered example the higher curvature terms significantly modify the post-collision spacetime. It appears that the pure Einstein gravity and the CTS argument based on it cannot be trusted in the future of the collision plane.

\section{FINAL REMARKS}

In this paper I questioned the use of classical Einstein gravity to analyze black hole production processes in transplanckian particle collisions. My basic argument was very

simple: I pointed out that curvature, as measured by the curvature invariant $\left(R_{\mu \nu \lambda \sigma}\right)^{2}$, becomes large on the transverse plane $u=v=0$ in the classical collision spacetime, in the region relevant for the horizon formation. 
In principle, I could have stopped right here, since this fact alone already implies that quantum corrections cannot be ignored. ${ }^{10}$ However, I decided to go a bit further and give a concrete example of how taking quantum corrections into account may dramatically change the picture.

Needless to say, the precise form of quantum corrections to classical general relativity remains so far unknown. However, there is a common opinion (supported by string theory) that at least to some extent these corrections may be represented by adding to the effective action of gravity a sequence of higher curvature terms.

In this paper we considered a particular example of such 'higher curvature gravity' - the so-called Einstein-Lovelock theory. We re-analyzed the collision in this theory and found that the spacetime does deviate significantly from the pure Einstein case. Thus, the initial conclusions are supported.

Of course, once it is found that a certain combination of higher curvature terms strongly modifies the Einstein gravity result, Pandora's box is open, and there is no reason to expect that all other higher curvature terms can be excluded from the analysis. Moreover, since the width of the colliding shock waves is $\sim E^{-1} \ll 1$, it seems very likely that the higher the power of curvature, the more important this term will become. (It was not so for the higher Lovelock Lagrangians solely because of antisymmetrization.)

For this reason, the given analysis of the Einstein-Lovelock gravity case should not perhaps be assigned importance beyond that of an example supporting the main argument. (Nevertheless, it seems to be the first study of a dynamical process in this theory; as far as we know, all previous applications involved static solutions.) The real conclusion of this analysis and the whole paper is that, at the present state of knowledge about quantum gravity, no well-founded claims about transplanckian collisions at small impact parameters can be made. Even if TeV-scale gravity is realized in nature, the true story of such collisions will be much more complicated than a single large black hole production with a subsequent Hawking evaporation.

${ }^{10}$ Perhaps some readers will find the following additional explanation useful. It is sometimes stated that quantum gravity corrections in transplanckian collisions should be small because the Schwarzschild radius $R_{\text {Schw }}(E) \gg 1$ for $E \gg 1$. This would indeed be true were $R_{\text {Schw }}(E)$ the only relevant length scale in the problem. But my analysis shows that it is not: the other relevant scale is set by the curvature radius near $u=v=0$ at $r \sim R_{\text {Schw }}(E)$, which according to (27) is actually much smaller than the Planck length. 


\section{Acknowledgments}

I would like to thank J. de Boer, L. Cornalba, D. Labutin, A. Naqvi, A. Polyakov, A. Sinkovics, K. Skenderis, M. Taylor, and the anonimous referee for many useful suggestions. This work was supported by Stichting FOM.

[1] N. Arkani-Hamed, S. Dimopoulos and G. R. Dvali, "The hierarchy problem and new dimensions at a millimeter," Phys. Lett. B 429, 263 (1998) arXiv:hep-ph/9803315;

I. Antoniadis, N. Arkani-Hamed, S. Dimopoulos and G. R. Dvali, "New dimensions at a millimeter to a Fermi and superstrings at a TeV," ibid. 436, 257 (1998) arXiv:hep-ph/9804398;

[2] V. M. Abazov et al. [D0 Collaboration], "Search for large extra dimensions in the monojet + missing-E(T) channel at D0," Phys. Rev. Lett. 90, 251802 (2003) arXiv:hep-ex/0302014.

[3] K. Hagiwara et al. [Particle Data Group Collaboration], "Review Of Particle Physics," Phys. Rev. D 66, 010001 (2002).

[4] M. Cavaglia, "Black hole and brane production in TeV gravity: A review," Int. J. Mod. Phys. A 18, 1843 (2003) arXiv:hep-ph/0210296;

G. Landsberg, "Black holes at future colliders and beyond: A review," in Proceedings of SUSY 2002 Conference, DESY Hamburg arXiv:hep-ph/0211043;

P. Kanti, "Black holes in theories with large extra dimensions: A review," arXiv:hep-ph/0402168

[5] S. Dimopoulos and G. Landsberg, "Black holes at the LHC," Phys. Rev. Lett. 87, 161602 (2001) arXiv:hep-ph/0106295;

S. B. Giddings and S. Thomas, "High energy colliders as black hole factories: The end of short distance physics," Phys. Rev. D 65, 056010 (2002) arXiv:hep-ph/0106219.

[6] G. Veneziano, "Quantum string gravity near the Planck scale," in Proceedings of the 5th seminar on Quantum Gravity, Moscow, 1990, edited by M. A. Markov, V. A. Berezin and V. P. Frolov (World Scientific, Singapore, 1991);

D. Amati, M. Ciafaloni and G. Veneziano, "Can Space-Time Be Probed Below The String Size?," Phys. Lett. B 216, 41 (1989); "Effective action and all order gravitational eikonal at Planckian energies," Nucl. Phys. B 403, 707 (1993); 
M. Fabbrichesi, R. Pettorino, G. Veneziano and G. A. Vilkovisky, "Planckian energy scattering and surface terms in the gravitational action," Nucl. Phys. B 419, 147 (1994) arXiv:hep-th/9309037.

[7] T. Banks and W. Fischler, "A model for high energy scattering in quantum gravity," arXiv:hep-th/9906038

[8] D. M. Eardley and S. B. Giddings, "Classical black hole production in high-energy collisions," Phys. Rev. D 66, 044011 (2002) arXiv:gr-qc/0201034.

[9] M. B. Voloshin, "Semiclassical suppression of black hole production in particle collisions," Phys. Lett. B 518, 137 (2001) arXiv:hep-ph/0107119; "More remarks on suppression of large black hole production in particle collisions," Phys. Lett. B 524, 376 (2002) arXiv:hep-ph/0111099.

[10] S. N. Solodukhin, "Classical and quantum cross-section for black hole production in particle collisions," Phys. Lett. B 533, 153 (2002) arXiv:hep-ph/0201248;

K. Krasnov, " $\Lambda<0$ quantum gravity in $2+1$ dimensions. II: Black hole creation by point particles," Class. Quant. Grav. 19, 3999 (2002) arXiv:hep-th/0202117;

S. D. H. Hsu, "Quantum production of black holes," Phys. Lett. B 555, 92 (2003) arXiv:hep-ph/0203154;

A. Jevicki and J. Thaler, "Dynamics of black hole formation in an exactly solvable model," Phys. Rev. D 66, 024041 (2002) arXiv:hep-th/0203172.

[11] D. Lovelock, "The Einstein Tensor And Its Generalizations," J. Math. Phys. 12, 498 (1971).

[12] R. Penrose, unpublished (1974), as discussed in P. D. D'Eath and P. N. Payne, Phys. Rev. D 46, 658 (1992).

[13] P. C. Aichelburg and R. U. Sexl, "On The Gravitational Field Of A Massless Particle," Gen. Rel. Grav. 2, 303 (1971).

[14] P. D. D'Eath, "High Speed Black Hole Encounters And Gravitational Radiation," Phys. Rev. D 18, 990 (1978).

[15] T. Dray and G. 't Hooft, "The Gravitational Shock Wave Of A Massless Particle," Nucl. Phys. B 253, 173 (1985).

[16] S.W. Hawking and G.F.R. Ellis, The Large Scale Structure of Space-Time (Cambridge University Press, 1973).

[17] H. Yoshino and Y. Nambu, "Black hole formation in the grazing collision of high-energy 
particles," Phys. Rev. D 67, 024009 (2003) arXiv:gr-qc/0209003.

[18] S. Deser, "Infinities in quantum gravities," Annalen Phys. 9, 299 (2000) arXiv:gr-qc/9911073.

[19] M. B. Green, J. H. Schwarz, and E. Witten, Superstring Theory. Vols. 1,2 (Cambridge University Press, 1987); J. Polchinski, String Theory. Vols. 1,2 (Cambridge University Press, 1998).

[20] C. Rovelli, "Loop quantum gravity," Living Rev. Rel. 1, 1 (1998) arXiv:gr-qc/9710008;

M. Gaul and C. Rovelli, "Loop quantum gravity and the meaning of diffeomorphism invariance," Lect. Notes Phys. 541, 277 (2000) arXiv:gr-qc/9910079.

[21] N. D. Birrell and P. C. Davies, Quantum Fields In Curved Space (Cambridge University Press, 1982).

[22] F. A. E. Pirani, "Gravitational Waves in General Relativity. IV. The Gravitational Field of a Fast-Moving Particle," Proc. Roy. Soc. Lond. A 252, 96 (1959).

[23] V. B. Berestetsky, E. M. Lifshitz and L. P. Pitaevsky, Quantum Electrodynamics (Pergamon, Oxford, 1982).

[24] O. V. Kancheli, "Parton picture of inelastic collisions at transplanckian energies," arXiv:hep-ph/0208021.

[25] R. C. Myers, "Black holes in higher curvature gravity," in Black Holes, Gravitational Radiation and the Universe, Essays in Honor of C.V. Vishveshwara, edited by B. R. Iyer, B. Bhawal, C.V. Vishveshwara (Kluwer Academic Publishers, 1998), p. 121 arXiv:gr-qc/9811042.

[26] N. Deruelle and J. Madore, “On the quasi-linearity of the Einstein- 'Gauss-Bonnet' gravity field equations," in Proceedings of the Conference in Honor of Yvonne Choquet-Bruhat, Cargese, 2002 arXiv:gr-qc/0305004.

[27] A. Barrau, J. Grain, and S. O. Alexeyev, "Gauss-Bonnet black holes at the LHC: Beyond the dimensionality of space," arXiv:hep-ph/0311238

[28] T. Eguchi, P. B. Gilkey, and A. J. Hanson, "Gravitation, Gauge Theories And Differential Geometry," Phys. Rept. 66, 213 (1980), p. 316;

S. Kobayashi and K. Nomizu, Foundations of Differential Geometry (Interscience, New York, 1969), Vol. 2, p. 318.

[29] C. C. Briggs, "A general expression for the quartic Lovelock tensor," arXiv:gr-qc/9703074,

F. Müller-Hoissen, "Spontaneous Compactification With Quadratic And Cubic Curvature Terms," Phys. Lett. B 163, 106 (1985). 
[30] C. Lanczos, "Elektromagnetismus als natürliche Eigenschaft der Riemannschen Geometrie," Z. Phys. 73, 147 (1932); "A Remarkable Property of the Riemann-Christoffel Tensor in Four Dimensions," Ann. Math. 39, 842 (1938).

[31] B. Zwiebach, "Curvature Squared Terms And String Theories," Phys. Lett. B 156, 315 (1985).

[32] K. S. Stelle, "Renormalization Of Higher Derivative Quantum Gravity," Phys. Rev. D 16, 953 (1977).

[33] R. R. Metsaev and A. A. Tseytlin, "Two Loop Beta Function For The Generalized Bosonic Sigma Model," Phys. Lett. B 191, 354 (1987).

[34] S. Deser and A. N. Redlich, "String Induced Gravity And Ghost Freedom," Phys. Lett. B 176, 350 (1986) [Erratum ibid. 186, 461 (1987)].

[35] C. P. Burgess, "Quantum gravity in everyday life: General relativity as an effective field theory," arXiv:gr-qc/0311082.

[36] J. B. Griffiths, Colliding Plane Waves In General Relativity (Oxford University Press, 1991).

[37] Y. Nutku and Ö. Sarioğlu, "An integrable family of Monge-Ampère equations and their multiHamiltonian structure," Phys. Lett. A 173, 270 (1993).

[38] R. Courant and D. Hilbert, Methods of Mathematical Physics (Interscience, New York, 1962), Vol. 2.

[39] A. M. Ignatov and V. P. Poponin, "Pulse interaction in nonlinear vacuum electrodynamics," arXiv:hep-th/0008021

[40] P.D. Lax, Hyperbolic Systems of Conservation Laws and the Mathematical Theory of Shock Waves (SIAM, Philadelphia, 1973).

[41] O.I. Mokhov and Y. Nutku, "Bianchi transformation between the real hyperbolic MongeAmpere equation and the Born-Infeld equation," Lett. Math. Phys. 32, 121 (1994).

[42] M. Arik, F. Neyzi, Y. Nutku, P.J. Olver, and J. M. Verosky, "Multi-Hamiltonian structure of the Born-Infeld equation," J. Math. Phys. 30, 1338 (1989). 